\title{
Editorial: Continuous Quality Improvement (CQI)-Advancing Understanding of Design, Application, Impact, and Evaluation of CQI Approaches
}

\author{
Ross Bailie ${ }^{1 *}$, Jodie Bailie ${ }^{1}$, Sarah Larkins ${ }^{2}$ and Edward Broughton ${ }^{3}$ \\ ${ }^{1}$ The University of Sydney, The University Centre for Rural Health, Lismore, NSW, Australia, ${ }^{2}$ James Cook University, College \\ of Medicine and Dentistry, Townsville, QLD, Australia, ${ }^{3}$ University Research Co., LLC, Chevy Chase, MD, United States
}

Keywords: primary health care, health systems research, continuous quality improvement, Aboriginal and Torres Strait Islander health, building block

\section{Editorial on the Research Topic}

Continuous Quality Improvement (CQI)-Advancing Understanding of Design, Application, Impact, and Evaluation of CQI Approaches

Continuous quality improvement (CQI) approaches are increasingly used to bridge gaps between the evidence base for best practice, what actually happens in practice, and achievement of better population health outcomes. Among a range of quality improvement strategies, CQI is characterized by iterative use of processes to identify quality problems, develop solutions, and implement and evaluate changes. Application of CQI in health care is evolving and evidence of their success continues to emerge (1-3).

Through the Research Topic, "Continuous Quality Improvement (CQI)-Advancing Understanding of Design, Application, Impact, and Evaluation of CQI approaches," we aimed to aggregate knowledge of useful approaches to tailoring CQI approaches for different contexts, and for implementation, scale-up and evaluation of CQI interventions/programs. This Research Topic has attracted seven original research reports and three "perspectives" papers. Thirty-six authors have contributed from eighteen research organizations, universities, and policy and service delivery organizations. All original research articles and one perspective paper come from the Australian Audit and Best Practice for Chronic Disease (ABCD) National Research Partnership ("ABCD Partnership") in Indigenous primary healthcare settings (4-6). To some extent, this reflects the interests and connections of two of the Topic Editors, who were lead investigators on the ABCD Partnership. This Partnership has made a prominent contribution to original research on CQI in primary healthcare internationally, with over 50 papers published in the peer-reviewed literature over the past 10 years.

As most articles in this Research Topic arise from the ABCD Partnership, a brief overview of the program provides a useful backdrop. The program originated in 2002 in the Top End of the Northern Territory in Australia, and built on substantial prior research and evaluation of CQI methods in Indigenous primary healthcare. With substantial growth and enthusiastic support from service providers and researchers around Australia, the ABCD Partnership has focused since 2010

Abbreviations: $\mathrm{ABCD}$, audit and best practice for chronic disease; CQI, continuous quality improvement. 
on exploring clinical performance variation, examining strategies for improving primary care, and working with health service staff, management and policy makers to enhance effective implementation of successful strategies (4). By the end of 2014, the ABCD Partnership had generated the largest and most comprehensive dataset on quality of care in Australian Indigenous primary healthcare settings. The Partnership's work is being extended through the Centre of Research Excellence in Integrated Quality Improvement (6).

Several research papers included in this Research Topic illustrate consistent findings of wide variation in adherence to clinical best-practice guidelines between health centers (Bailie et al.; Burnett et al.; Matthews et al.). The papers also show variation among different aspects of care, with relatively good delivery of some modes of care [Bailie et al.; (7)] and poor delivery of others-such as follow-up of abnormal clinical or laboratory findings. These findings are evident in eye care (Burnett et al.), general preventive clinical care (Bailie et al.), and in absolute cardiovascular risk assessment (Matthews et al.; Vasant et al.). The findings are consistent with other $\mathrm{ABCD}$-related publications on diabetes care (8), preventive health (9), maternal care (10), child health (11), rheumatic heart disease (12), and sexual health (13).

Systems to support good clinical care are explored by Woods et al. in five primary healthcare centers that were identified through $\mathrm{ABCD}$ data as achieving substantially greater improvement than others over successive CQI cycles. Attention to understanding and improving systems was shown to be vital to the improvements in clinical care achieved by these health centers. Improved staffing and commitment to working in the community were standout aspects of health center systems that underpinned improvements in clinical care.

On a wider scale, engagement by primary healthcare services in the ABCD Partnership has enabled assessment of system functioning at district, regional, state, and national levels, as reflected in stakeholders' perceptions of barriers and enablers to addressing gaps in chronic illness care and child health, and identifying drivers for improvement (Bailie et al.). Primary drivers included staff capability, availability and use of information systems and decision support tools, embedding of CQI processes, and community engagement. We have also shown how consistent and sustained policy and infrastructure support for CQI enables large-scale and ongoing improvements in quality of care (3).

Commitment of the ABCD team to promoting effective use of CQI data is reflected in one "perspective" paper, which describes a theory-informed cyclical interactive dissemination strategy (Laycock et al.). Concurrent developmental evaluation provides a mechanism for learning and refinement over successive cycles (14).

The other two perspective articles (not specifically from the ABCD program) highlight the role of facilitation in CQI and the potential for application of CQI in health professional education. The emerging evidence on facilitation as a vital tool for effective CQI should guide resourcing and approaches to CQI (Harvey and Lynch). The approach builds on the humanistic principles of modern CQI methods-participation, engagement, shared decision-making, enabling others, and tailoring to context. The framework for CQI approaches to health professional education described by Clithero et al. directly addresses a critical need for innovative approaches to health workforce development that will strengthen community engagement and embed CQI principles into health system functioning. The scale and scope of need in workforce development is strongly evident in findings of the ABCD program.

Importantly, CQI methods are proving useful in assessing and potentially improving delivery of evidence-based health promotion practices (Percival et al.). Percival's experience in this field highlights the health facility and wider system challenges facing effective implementation of CQI methods. In health promotion these barriers include low priority given to health promotion in the face of heavy demands for acute clinical care. This work in health promotion complements other research on applying CQI to social determinants of health more broadly (15), including community food supply (16), housing (17), and education (18).

The publications in this special issue address many of the "building blocks" of high performing primary care described by Bodenheimer and colleagues in the US; namely, four foundational components (engaged leadership, data-driven improvement, empanelment, and team-based care) that are vital to facilitate the implementation of the other six elements (patient-team partnership, population management, continuity of care, prompt access to care, comprehensiveness, and care coordination) (19). They are also relevant to Australian based work on clinical microsystems and development of CQI tools for mainstream general practice, such as the Primary Care-Practice Improvement Tool (with similar components to the ABCD systems assessment tool) (20).

Continuous quality improvement is vital to improving health outcomes through system strengthening. We anticipate substantial future development of CQI methods. By late 2017, there had been over 20,000 views of this Research Topic, and many articles have already been cited in peer-review manuscripts. Further research on CQI in primary healthcare would be well guided by a systematic scoping review of literature summarizing empirical research on current knowledge in the field, and identifying key knowledge gaps.

\section{AUTHOR CONTRIBUTIONS}

RB wrote the first draft. JB has revised content and structure. SL and $\mathrm{EB}$ reviewed and edited subsequent drafts. All authors have approved the final version of the manuscript for publication.

\section{ACKNOWLEDGMENTS}

We would like to thank all of those who contributed to this Research Topic as authors, review editors, and colleagues.

\section{FUNDING}

The National Health and Medical Research Council funded the ABCD National Research Partnership Project (grant number 545267) and the Centre for Research Excellence in Integrated Quality Improvement (grant number 1078927). In-kind and financial support was provided by the Lowitja Institute and a range of Community-Controlled and Government agencies. 


\section{REFERENCES}

1. Tricco AC, Ivers NM, Grimshaw JM, Moher D, Turner L, Galipeau J, et al. Effectiveness of quality improvement strategies on the management of diabetes: a systematic review and meta-analysis. Lancet (2012) 379(9833):2252-61. doi:10.1016/S0140-6736(12)60480-2

2. Lewin S, Lavis JN, Oxman AD, Bastias G, Chopra M, Ciapponi A, et al. Supporting the delivery of cost-effective interventions in primary healthcare systems in low-income and middle-income countries: an overview of systematic reviews. Lancet (2008) 372(9642):928-39. doi:10.1016/ S0140-6736(08)61403-8

3. Bailie R, Matthews V, Larkins S, Thompson S, Burgess P, Weeramanthri T, et al. Impact of policy support on uptake of evidence-based continuous quality improvement activities and the quality of care for Indigenous Australians: a comparative case study. BMJ Open (2017) 7(10). doi:10.1136/ bmjopen-2017-016626

4. Bailie R, Si D, Shannon C, Semmens J, Rowley K, Scrimgeour DJ, et al. Study protocol: national research partnership to improve primary health care performance and outcomes for Indigenous peoples. BMC Health Serv Res (2010) 10:129. doi:10.1186/1472-6963-10-129

5. Bailie R, Matthews V, Brands J, Schierhout G. A systems-based partnership learning model for strengthening primary healthcare. Implement Sci (2013) 8(1):143. doi:10.1186/1748-5908-8-143

6. Bailie J, Schierhout G, Cunningham F, Yule J, Laycock A, Bailie R. Quality of primary health care for Aboriginal and Torres Strait Islander People in Australia: key research findings and messages for action from the $A B C D$ National Research Partnership. Menzies Sch Health Res (2015). doi:10.13140/ RG.2.1.3887.2801

7. Schierhout G, Matthews V, Connors C, Thompson S, Kwedza R, Kennedy C, et al. Improvement in delivery of type 2 diabetes services differs by mode of care: a retrospective longitudinal analysis in the Aboriginal and Torres Strait Islander primary health care setting. BMC Health Serv Res (2016) 16(1):560. doi:10.1186/s12913-016-1812-9

8. Matthews V, Schierhout G, McBroom J, Connors C, Kennedy C, Kwedza R, et al. Duration of participation in continuous quality improvement: a key factor explaining improved delivery of type 2 diabetes services. BMC Health Serv Res (2014) 14(1):578. doi:10.1186/s12913-014-0578-1

9. Bailie J, Matthews V, Laycock A, Schultz R, Burgess CP, Peiris D, et al. Improving preventive health care in Aboriginal and Torres Strait Islander primary care settings. Global Health (2017) 13(1):48. doi:10.1186/s12992-017-0267-z

10. Gibson-Helm ME, Teede HJ, Rumbold AR, Ranasinha S, Bailie RS, Boyle JA. Continuous quality improvement and metabolic screening during pregnancy at primary health centres attended by Aboriginal and Torres Strait Islander women. Med J Aust (2015) 203(9):369-70. doi:10.5694/mja14.01660

11. McAullay D, McAuley K, Bailie R, Mathews V, Jacoby P, Gardner K, et al. Sustained participation in annual continuous quality improvement activities improves quality of care for Aboriginal and Torres Strait Islander children. J Paediatr Child Health (2017). doi:10.1111/jpc.13673

12. Ralph AP, Fittock M, Schultz R, Thompson D, Dowden M, Clemens T, et al. Improvement in rheumatic fever and rheumatic heart disease management and prevention using a health centre-based continuous quality improvement approach. BMC Health Serv Res (2013) 13(1):525. doi:10.1186/ 1472-6963-13-525

13. Nattabi B, Matthews V, Bailie J, Rumbold A, Scrimgeour D, Schierhout G, et al. Wide variation in sexually transmitted infection testing and counselling at aboriginal primary health care centres in Australia: analysis of longitudinal continuous quality improvement data. BMC Infect Dis (2017) 17:148. doi:10.1186/s12879-017-2241-z

14. Laycock A, Bailie J, Matthews V, Cunningham F, Harvey G, Percival N, et al. A developmental evaluation to enhance stakeholder engagement in a wide-scale interactive project disseminating quality improvement data: study protocol for a mixed-methods study. BMJ Open (2017) 7:7. doi:10.1136/ bmjopen-2017-016341

15. McDonald EL, Bailie R, Michel T. Development and trialling of a tool to support a systems approach to improve social determinants of health in rural and remote Australian communities: the healthy community assessment tool. Int J Equity Health (2013) 12(1):15. doi:10.1186/1475-9276-12-15

16. Brimblecombe J, van den Boogaard C, Wood B, Liberato SC, Brown J, Barnes A, et al. Development of the good food planning tool: a food system approach to food security in Indigenous Australian remote communities. Health Place (2015) 34:54-62. doi:10.1016/j.healthplace.2015.03.006

17. Bailie RS, Wayte KJ. A continuous quality improvement approach to indigenous housing and health. Environ Health (2006) 6(2):36-41.

18. McCalman J, Bainbridge R, Russo S, Rutherford K, Tsey K, Wenitong M, et al. Psycho-social resilience, vulnerability and suicide prevention: impact evaluation of a mentoring approach to modify suicide risk for remote Indigenous Australian students at boarding school. BMC Public Health (2016) 16(1):98. doi:10.1186/s12889-016-2762-1

19. Bodenheimer T, Ghorob A, Willard-Grace R, Grumbach K. The 10 building blocks of high-performing primary care. Ann Fam Med (2014) 12(2):166-71. doi:10.1370/afm.1616

20. Crossland L, Janamian T, Sheehan M, Siskind V, Hepworth J, Jackson CL. Development and pilot study of the primary care practice improvement tool (PC-PIT): an innovative approach. Med J Aust (2014) 201(3):S52-5. doi:10.5694/mja14.00262

Conflict of Interest Statement: The authors declare that the research was conducted in the absence of any commercial or financial relationships that could be construed as a potential conflict of interest. RB was the chief investigator on the ABCD National Research Partnership and is the chief investigator on the Centre of Research Excellence in Integrated Quality Improvement. All papers published in the Research Topic received peer review from members of the Frontiers in Public Health Policy panel of reviewers who were independent of named authors on any given article published in this volume, consistent with the journal policy on conflict-of-interest.

Copyright $\odot 2017$ Bailie, Bailie, Larkins and Broughton. This is an open-access article distributed under the terms of the Creative Commons Attribution License (CC BY). The use, distribution or reproduction in other forums is permitted, provided the original author(s) or licensor are credited and that the original publication in this journal is cited, in accordance with accepted academic practice. No use, distribution or reproduction is permitted which does not comply with these terms. 